\title{
Diagnostic performance of CT-arthrography and 1.5T MR-arthrography for the assessment of glenohumeral joint cartilage: a comparative study with arthroscopic correlation
}

\author{
Patrick Omoumi • Alexandra Rubini • \\ Jean-Emile Dubuc • Bruno C. Vande Berg • \\ Frédéric E. Lecouvet
}

Received: 7 May 2014 / Revised: 13 August 2014 / Accepted: 15 October 2014 /Published online: 8 November 2014

(C) European Society of Radiology 2014

\begin{abstract}
Purpose To compare the diagnostic performance of multidetector CT arthrography (CTA) and 1.5-T MR arthrography (MRA) in detecting hyaline cartilage lesions of the shoulder, with arthroscopic correlation.

Patients and methods CTA and MRA prospectively obtained in 56 consecutive patients following the same arthrographic procedure were independently evaluated for glenohumeral cartilage lesions (modified Outerbridge grade $\geq 2$ and grade 4) by two musculoskeletal radiologists. The cartilage surface was divided in 18 anatomical areas. Arthroscopy was taken as the reference standard. Diagnostic performance of CTA and MRA was compared using ROC analysis. Interobserver and intraobserver agreement was determined by $\kappa$ statistics.

Results Sensitivity and specificity of CTA varied from 46.4 to $82.4 \%$ and from 89.0 to $95.9 \%$ respectively; sensitivity and specificity of MRA varied from 31.9 to $66.2 \%$ and from 91.1 to $97.5 \%$ respectively. Diagnostic performance of CTA was statistically significantly better than MRA for both readers (all $p \leq$ 0.04). Interobserver agreement for the evaluation of cartilage lesions was substantial with CTA $(\kappa=0.63)$ and moderate with MRA $(\kappa=0.54)$. Intraobserver agreement was almost perfect with both CTA $(\kappa=0.94-0.95)$ and MRA $(\kappa=0.83-0.87)$.
\end{abstract}

P. Omoumi $\cdot$ A. Rubini $\cdot$ B. C. Vande Berg $\cdot$ F. E. Lecouvet Department of Radiology, Cliniques Universitaires St Luc Université Catholique de Louvain, Hippocrate Avenue 10/2942, 1200 Brussels, Belgium

P. Omoumi $(\square)$

Department of Radiology, Lausanne University Hospital, Bugnon 46, 1011 Lausanne, Switzerland

e-mail: patrick.omoumi@chuv.ch

\section{J.-E. Dubuc}

Department of Orthopedic Surgery, Cliniques Universitaires St LucUniversité Catholique de Louvain, Hippocrate Avenue 10/2942, 1200 Brussels, Belgium
Conclusion The diagnostic performance of CTA and MRA for the detection of glenohumeral cartilage lesions is moderate, although statistically significantly better with CTA.

Key points

- CTA has moderate diagnostic performance for detecting glenohumeral cartilage substance loss.

- MRA has moderate diagnostic performance for detecting glenohumeral cartilage substance loss.

- CTA is more accurate than MRA for detecting cartilage substance loss.

Keywords Shoulder · Magnetic resonance imaging · Computed tomography $\cdot$ Arthrography $\cdot$ Cartilage

\section{Introduction}

Over the past few years, glenohumeral cartilage has raised interest in the radiology as well as the orthopaedic communities owing to the widespread use of imaging and arthroscopy, which have shown that cartilage lesions are more common in the shoulder than previously thought $[1,2]$. As an example, cartilage lesions are reported to be present in $29 \%$ of patients with subacromial impingement syndrome, with some arthroscopic series presenting cartilage lesions in more than $45 \%$ of cases [2-4]. The presence of cartilage lesions may impact the management and prognosis of shoulder pathology $[1,3]$. While treatment options for both focal and diffuse cartilage lesions are developed, the optimal imaging technique, which will allow not only diagnosis of these lesions but also help evaluate their implications on patient management and the efficacy of therapeutics, still needs to be defined [1].

Compared to magnetic resonance imaging (MRI) of other joints such as the knee, MRI of the glenohumeral cartilage is 
challenging. This is in part because of the relative thinness of glenohumeral cartilage, but also because of the configuration of the shoulder, away from the isocentre of most magnets and from the coils [5-7]. As a consequence, the diagnostic performance of MRI in the diagnosis of glenohumeral cartilage lesions is only moderate $[4,8]$.

Compared to MRI, magnetic resonance arthrography (MRA) and computed tomography arthrography (CTA) may have the potential to improve the visualization of cartilage lesions. Both benefit from the intra-articular injection of contrast material, while CTA has the advantage of a high spatial resolution as well as a high contrast between the hypodense cartilage and hyperdense surrounding bone and contrast material [9, 10].

Both MRA and CTA have proven to be valuable diagnostic tools for the evaluation of internal derangement of the shoulder, particularly in the preoperative setting [11-16]. Their accuracy has been previously compared in the same patients for the assessment of rotator cuff tears and shoulder instability lesions, with the diagnostic performance of CTA being similar or better compared to MRA $[11,17]$.

The purpose of this work was to compare the diagnostic performance of CTA and MRA in assessing the entire glenohumeral cartilage, by prospectively acquiring examinations with the two techniques in the same series of patients, with arthroscopic findings taken as a reference.

\section{Materials and methods}

\section{Patient population}

This study was approved by our institutional ethical committee and informed consent was obtained from all patients. Over a 6-month period, one orthopaedic surgeon prospectively included all consecutive patients who presented with chronic shoulder pain or shoulder instability requiring an arthroscopic treatment, based on clinical and imaging findings. Radiograph, ultrasound and/or conventional MRI had been obtained in all patients. The exclusion criteria were the presence of severe shoulder osteoarthritis (Kellgren-Lawrence 3 or 4 ) or history of previous shoulder surgery.

Fifty-six consecutive patients (mean age \pm standard deviation, $46 \pm 15$ years; range, $16-71$ years; 32 men) were prospectively enrolled. The final diagnoses, based on the analysis of arthroscopic as well as imaging findings, were rotator cuff tendon tears in $59 \%$ of patients $(n=33)$, rotator cuff tendinosis in $18 \%$ of patients $(n=10)$, rotator cuff calcifications in $14 \%$ of patients $(n=8)$ and labroligamentous instability lesions in $29 \%$ of patients $(n=16)$ (more than one diagnosis per patient was possible).

\section{CTA/MRA}

At our institution, preoperative CTA is routinely performed for patients who necessitate therapeutic shoulder arthroscopy to plan the procedure. For the purpose of this study, MRA was performed in addition to CTA in all patients, following a single intra-articular injection of a mixture of iodine and gadolinium-based contrast material. The average delay between CTA and MRA examinations was $20 \mathrm{~min}$.

The same radiologist performed all procedures. Following local anaesthesia, $15 \mathrm{~mL}$ of a mixture of $5 \mathrm{~mL}$ of ionic contrast material (meglumine ioxaglate and sodium ioxaglate, Hexabrix 320, Guerbet, Aulnay-sous-bois, France) and $20 \mathrm{~mL}$ of diluted gadopentetate dimeglumine (Artirem, Guerbet) (final concentration of $2.0 \mathrm{mmol} / \mathrm{L}$ ) was injected into the glenohumeral joint under fluoroscopic guidance, using an anterior approach $[3,11]$. After the injection procedure, gentle passive mobilization of the shoulder was performed to allow good diffusion of the contrast material in the joint cavity.

Patient position was the same for both imaging modalities: supine, head first, arm along the body, with the shoulder in neutral position.

Spiral CT was performed immediately after joint opacification on a 16-detector helical CT (MX 8000 IDT, Philips Medical Systems, Best, the Netherlands). Following a frontal projection scout image, a 10- to 15 -s acquisition was performed to image the volume from the top of the acromioclavicular joint to the lower margin of the axillary recess of the glenohumeral joint. Acquisition parameters were $120 \mathrm{kV}$ and $350 \mathrm{mAs}$, focal spot size, $0.8 \times 1.2 \mathrm{~mm}$; collimation beam, $12 \mathrm{~mm}$; FOV, 15-20 cm; effective pitch, 0.3; effective thickness, about $0.8 \mathrm{~mm} ; 512 \times 512$ matrix. Images were reconstructed using a 3D Cone Beam back projection algorithm, a high frequency kernel, an increment of $0.4 \mathrm{~mm}$ (50\% section overlap) and a zoom factor of 1.2.

Immediately following the CT examination, MRA was performed on a 1.5-T magnet (Gyroscan Intera; Philips Medical Systems), using a dedicated shoulder coil.

The imaging planes were as follows: transverse, coronal oblique and sagittal oblique (parallel to the glenoid) planes. Fat-suppressed T1- (TR/TE, 500/13 ms; 3 NSA; matrix, $256 \times 256(384 \times 512$ coronal $)$; acquisition times, 3'25' in the sagittal plane, 3'31" in the coronal plane and 2'53" in the transverse plane); proton density (PD) (TR/TE, 2,3502,450/20-25 ms; 3 NSA; ETL6 (coronal), ETL4 (axial); matrix, $256 \times 256$; acquisition times: $2{ }^{\prime} 26^{\prime \prime}$ in the coronal plane and 3'43' in the transverse plane) and T2-weighted images (TR/TE, 2,650/90 ms; ETL 6; matrix, 256×256; acquisition time, 3'10" in the sagittal oblique plane) were obtained. Slice thickness was $3 \mathrm{~mm}$ with 0.3 -mm gaps; the FOV was $160 \times 126 \mathrm{~mm}$. Total MR examination time ranged from 15 to $20 \mathrm{~min}$. 
Arthroscopy

Arthroscopy, considered as our reference standard, was performed at our institution by the same fellowship-trained orthopaedic surgeon (with 15 years of experience in shoulder arthroscopy), within 1 month after the preoperative imaging.

The articular cartilage was graded according to a modified Outerbridge and Noyes classification system, mainly based on the depth of substance loss [10, 18, 19]. The grading of cartilage lesions was performed in nine anatomic areas for both the humeral head and glenoid cavity, and reported in diagrams on which each articular surface was visually divided into three thirds in both cranio-caudal and anteroposterior directions (Fig. 1). For this study, we considered as abnormal all areas with chondral tissue loss (grade $\geq 2$ ). We also considered separately full-thickness cartilage defects (grade 4).

Other associated lesions, i.e. tears of the rotator cuff, capsulo-labral or other joint disorders were diagnosed and treated during the same arthroscopic procedure.

Image analysis

All CTA and MRA studies were prospectively stored on our institution's PACS, on which the image analysis was performed.

CTA and MRA were analysed independently by two fellowship-trained radiologists (10 and 4 years of experience in musculoskeletal radiology), both unaware of findings at arthroscopy or any other imaging examination. MRA and CTA examinations were reviewed in a random order, blinded to the patient identification. One observer repeated the readings after a 3-month period to assess the intraobserver agreement.

For MRA, all sequences were used for the analysis. For CTA, coronal oblique, sagittal oblique and axial multiplanar reformats (MPR) were used, with a $0.8-\mathrm{mm}$ section thickness,
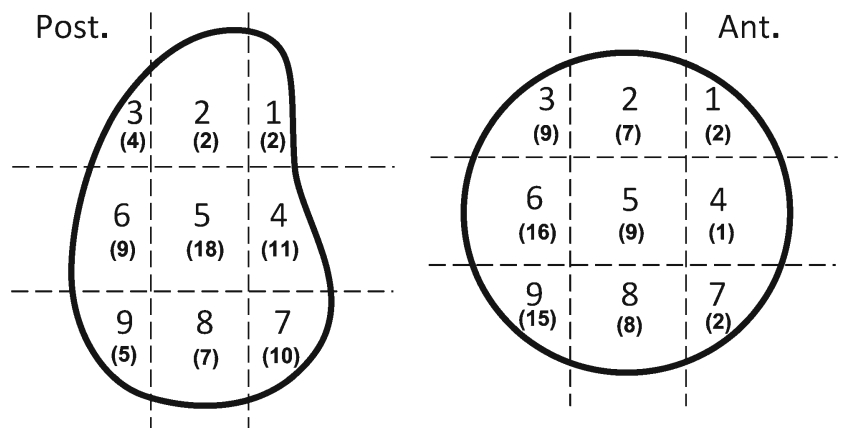

Fig. 1 Schematic view of the delimitation of nine anatomic regions in each humeral head (right) and glenoid (left) cartilage surface. These surfaces are divided into three virtual thirds in both cranio-caudal and anteroposterior directions. These diagrams were used for cartilage grades with arthroscopy as well as CTA and MRA. Numbers in parentheses correspond to number of cartilage lesions (grade $\geq 2$ ) in each subarea diagnosed by arthroscopy and bone windowing presets (window width, 1,900 HU; window level, $450 \mathrm{HU})$.

The cartilage surface was divided into nine anatomical areas in the same fashion as for arthroscopy (Fig. 1). The cartilage in each area was graded as follows: "normal" when no clear penetration of contrast material was observed (Outerbridge grade $\leq 1$ ), "abnormal" when there was penetration of contrast material into the cartilage (Outerbridge grade $\geq 2$ ) (Figs. 2, 3, 4 and 5).

\section{Statistical analysis}

Sensitivity and specificity of CTA and MRA for the detection of cartilage lesions were calculated for each imaging technique. The diagnostic performance was successively evaluated when considering all areas with abnormal cartilage (grade $\geq 2$ ), and for each glenoidal and humeral cartilage area separately. The analysis was repeated with grade 4 lesions only. The diagnostic performances were compared using pairwise comparisons of ROC curves [20-22]. A significance level of $p=0.05$ was considered for all tests.

Inter- and intraobserver agreement for all grades was assessed by $\mathrm{k}$ statistics. As suggested by Landis and Koch, $\kappa$ values were considered to represent poor agreement when less than 0 ; slight agreement between 0 and 0.2 ; fair agreement between 0.21 and 0.40 ; moderate agreement between 0.41 and 0.60 ; substantial agreement between 0.61 and 0.80 ; and almost perfect agreement between 0.81 and 1.0 [23].

\section{Results}

\section{Arthroscopy}

In total, $57 \%(n=32)$ of patients had at least one grade $\geq 2$ lesions in the shoulder, $50 \%(n=28)$ at least one grade $\geq 2$ lesions on the glenoid and $45 \%(n=25)$ at least one grade $\geq 2$ lesions in the humeral head.

A total of 1,008 articular areas (504 humeral and 504 glenoidal areas) were graded in 56 patients. Thus, $65 \%$ $(n=656)$ of surface areas (from 51 shoulders) were intact (grade 0$) ; 21 \%(n=215)$ (from 36 shoulders) were categorized as grade $1,5 \%(n=51)$ (from 24 shoulders) as grade $2,3 \%$ $(n=31)$ (from 20 shoulders) as grade 3 and $5 \%(n=55)$ (from 17 shoulders) as grade 4 .

Three patients (all with shoulder instability) underwent specific treatment of cartilage lesions (two debridements and one microfracture) in addition to the arthroscopic treatment of the underlying conditions. 
Fig. 2 Fifty-one-year-old man with grade 2 cartilage lesion at anterior aspect of glenoid (arrows) at arthroscopy, correctly diagnosed by both readers at CTA (a) and MRA (b). a CTA with axial reformats. b MRA with fatsuppressed T1-weighted SE sequence
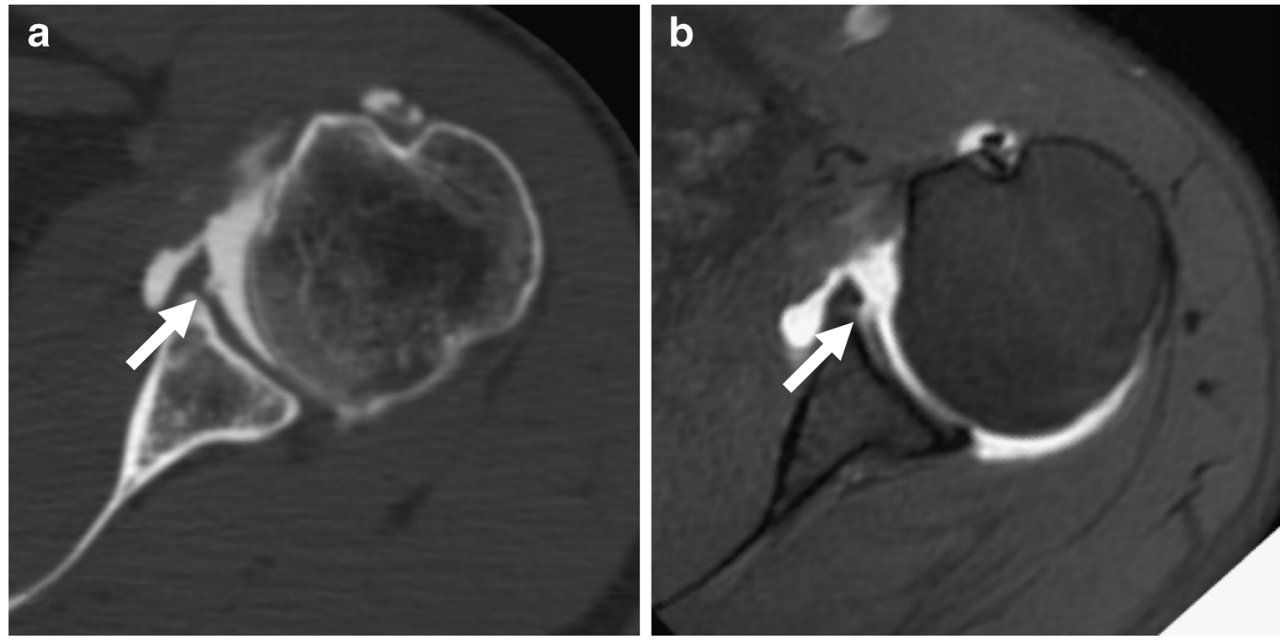

Diagnostic performance of CTA and MRA for the evaluation of cartilage lesions

Detailed sensitivities, specificities and accuracies of CTA and MRA in detecting cartilage lesions are reported in Table 1 (grade $\geq 2$ ) and Table 2 (grade 4) and illustrated in Fig. 6.

When considering all cartilage lesions with substance loss (grade $\geq 2$ ), sensitivity and specificity of CTA varied from 46.4 to $82.4 \%$ and from 89.0 to $95.9 \%$ respectively; sensitivity and specificity of MRA varied from 31.9 to $66.2 \%$ and from 91.1 to $97.5 \%$ respectively.

Diagnostic performance of CTA was statistically significantly better than MRA for both readers, and both glenoidal and humeral areas $(p \leq 0.04)$.

When comparing the diagnostic performance between cartilage surfaces (humeral head vs. glenoid), both CTA and MRA performed statistically significantly better for the glenoid cartilage than the humeral head for reader 2 $(p<0.02)$. For reader 1 , the diagnostic performance was better for the glenoid cartilage than the humeral head for both techniques, but statistical significance was not reached ( $p=0.29$ for CTA and $p=0.17$ for MRA).
When considering grade 4 cartilage lesions only, sensitivity and specificity of CTA varied from 48.3 to $84.6 \%$ and from 99.0 to $99.8 \%$ respectively; sensitivity and specificity of MRA varied from 17.2 to $76.9 \%$ and from 99.4 to $99.8 \%$ respectively.

Diagnostic performance of CTA was better than MRA for all comparisons, but only reached statistical significance for the evaluation of all cartilage areas, as well as humeral head cartilage lesions for reader 1 (both $p<0.01$, all other $p \geq 0.05)$.

When comparing the diagnostic performance between cartilage surfaces (humeral head vs. glenoid), MRA performed statistically significantly better for the glenoid cartilage areas than the humeral head areas for both readers $(p<0.01)$. With CTA, the diagnostic performance was better for the glenoid cartilage than the humeral head, but statistical significance was not reached $(p=0.06$ for reader 1 and $p=0.15$ for reader 2 ).

Inter- and intraobserver agreement

Interobserver agreement for the evaluation of cartilage lesions (grade $\geq 2)$ was substantial with CTA $(\kappa=0.63)$ and moderate
Fig. 3 Forty-seven-year-old man with grade 2 cartilage lesions at posterior aspect of glenoid at arthroscopy, correctly diagnosed at CTA (a) (white arrow) but interpreted as normal by both readers at MRA (b). a CTA with axial reformats. b MRA with fatsuppressed T1-weighted SE sequence
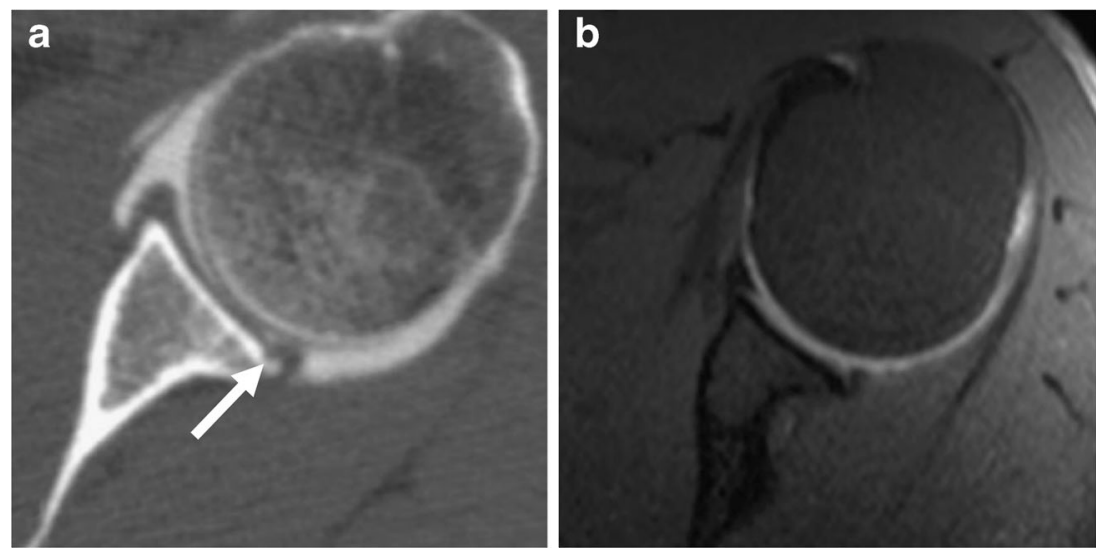
Fig. 4 Sixty-two-year-old woman with grade 4 cartilage lesion on glenoid (arrows) and grade 3 cartilage lesion on humeral head (open arrows) at arthroscopy, correctly diagnosed by both readers at CTA (a and b) and MRA (c and d). CTA with axial (a) and coronal oblique (b) reformats. MRA with axial (c) and coronal oblique (d) fatsuppressed T1-weighted SE sequences
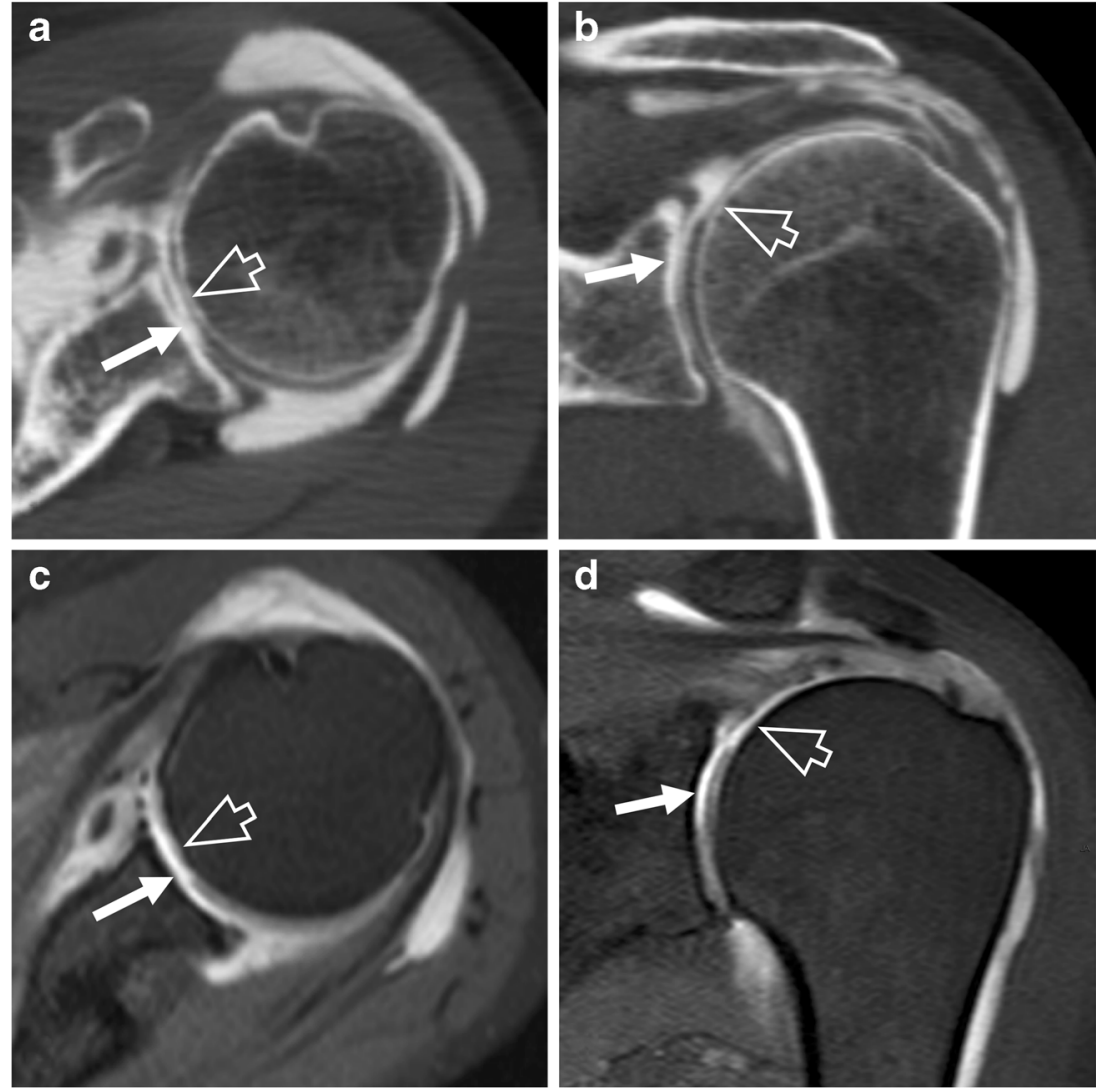

with MRA $(\kappa=0.54)$ (Table 3$)$. Interobserver agreement was higher for the glenoidal cartilage areas compared to the humeral head areas ( $\kappa=0.68$ and 0.59 vs. 0.58 and 0.46 for CTA and MRA respectively). Intraobserver agreement was almost perfect with both CTA $(\kappa=0.94-0.95)$ and MRA $(\kappa=0.83-0.87)$.

\section{Discussion}

In this study, we showed that the diagnostic performance of both CTA and MRA in assessing glenohumeral cartilage lesions was moderate, although it was statistically significantly better with CTA.

The diagnostic performance of CTA and MRA for the evaluation of cartilage has been evaluated and compared in other joints, with most research focusing on the knee [24-26]. Few studies have focused on the thinner glenohumeral cartilage. Guntern et al. found "moderate performance" of MRA for the diagnosis of all types of cartilage lesions, with sensitivities and specificities varying from 53 to $100 \%$ and 51 to $87 \%$ respectively [3], while Hayes et al. found sensitivities and specificities ranging from 82.3 to $86.8 \%$ and 80.0 to $88.2 \%$, respectively [27]. However, these two studies on MRA were retrospective. Lecouvet et al. evaluated the
Fig. 5 Fifty-nine-year-old woman with grade 4 cartilage lesion at posterior margin of humeral head at arthroscopy, missed by both readers at CTA and MRA (arrows). a CTA with axial reformats. b MRA with fatsuppressed T1-weighted SE sequence
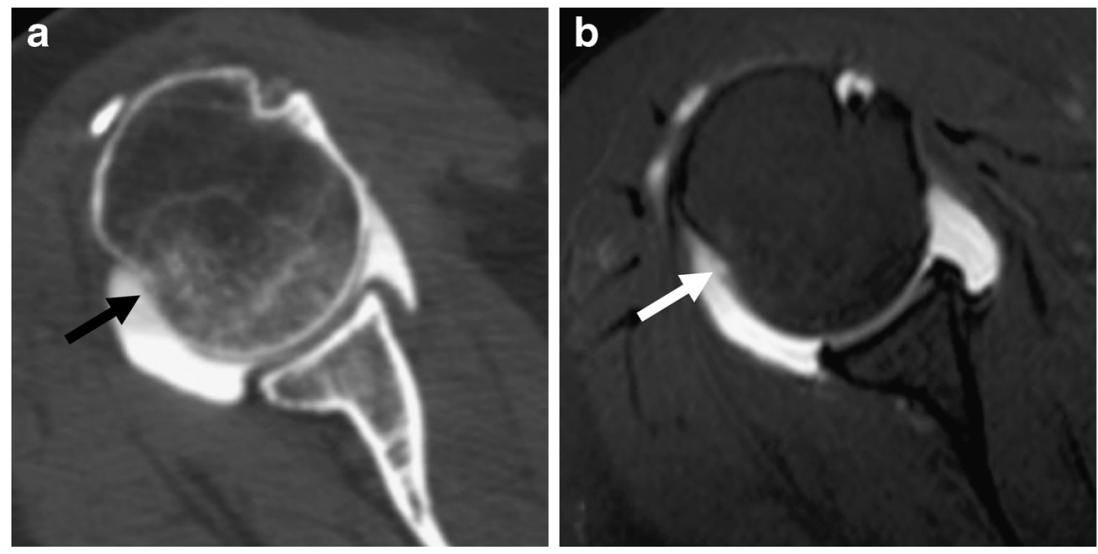
Table 1 Sensitivities, specificities and accuracy (\%) of CTA and MRA in detecting all cartilage lesions (grade $\geq 2$ )

\begin{tabular}{|c|c|c|c|c|c|}
\hline & & \multicolumn{2}{|l|}{ Reader 1} & \multicolumn{2}{|l|}{ Reader 2} \\
\hline & & CTA & MRA & CTA & MRA \\
\hline \multirow[t]{3}{*}{ All cartilage areas $(n=1,008)$} & Sensitivity & $\begin{array}{l}78.8 \\
{[71.0-85.3]}\end{array}$ & $\begin{array}{l}59.1 \\
{[50.4-67.4]}\end{array}$ & $\begin{array}{l}58.4 \\
{[49.7-66.7]}\end{array}$ & $\begin{array}{l}42.3 \\
{[33.9-51.1]}\end{array}$ \\
\hline & Specificity & $\begin{array}{l}95.5 \\
{[93.9-96.8]}\end{array}$ & $\begin{array}{l}96.2 \\
{[94.7-97.4]}\end{array}$ & $\begin{array}{l}89.6 \\
{[87.3-91.5]}\end{array}$ & $\begin{array}{l}91.5 \\
{[89.5-93.3]}\end{array}$ \\
\hline & Accuracy & $\begin{array}{l}93.3 \\
{[87.4-99.4]}\end{array}$ & $\begin{array}{l}91.2 \\
{[85.4-97.3]}\end{array}$ & $\begin{array}{l}85.3 \\
{[79.7-91.2]}\end{array}$ & $\begin{array}{l}84.8 \\
{[79.2-90.1]}\end{array}$ \\
\hline \multirow[t]{3}{*}{ Glenoidal cartilage areas $(n=504)$} & Sensitivity & $\begin{array}{l}82.4 \\
{[71.2-90.5]}\end{array}$ & $\begin{array}{l}66.2 \\
{[53.7-77.2]}\end{array}$ & $\begin{array}{l}70.6 \\
{[58.3-81.0]}\end{array}$ & $\begin{array}{l}52.9 \\
{[40.4-65.2]}\end{array}$ \\
\hline & Specificity & $\begin{array}{l}95.9 \\
{[93.6-97.5]}\end{array}$ & $\begin{array}{l}94.5 \\
{[92.5-96.8]}\end{array}$ & $\begin{array}{l}90.1 \\
{[86.9-92.8]}\end{array}$ & $\begin{array}{l}91.1 \\
{[88.0-93.6]}\end{array}$ \\
\hline & Accuracy & $\begin{array}{l}94.1 \\
{[85.8-99.9]}\end{array}$ & $\begin{array}{l}91.0 \\
{[82.9-99.8]}\end{array}$ & $\begin{array}{l}87.5 \\
{[79.5-96.1]}\end{array}$ & $\begin{array}{l}85.9 \\
{[78.0-94.4]}\end{array}$ \\
\hline \multirow[t]{3}{*}{ Humeral head cartilage areas $(n=504)$} & Sensitivity & $\begin{array}{l}75.4 \\
{[63.5-84.9]}\end{array}$ & $\begin{array}{l}52.2 \\
{[39.8-64.4]}\end{array}$ & $\begin{array}{l}46.4 \\
{[34.3-58.8]}\end{array}$ & $\begin{array}{l}31.9 \\
{[21.2-44.2]}\end{array}$ \\
\hline & Specificity & $\begin{array}{l}95.2 \\
{[92.7-97.0]}\end{array}$ & $\begin{array}{l}97.5 \\
{[95.5-98.7]}\end{array}$ & $\begin{array}{l}89.0 \\
{[85.6-91.8]}\end{array}$ & $\begin{array}{l}92.0 \\
{[89.0-94.3]}\end{array}$ \\
\hline & Accuracy & $\begin{array}{l}92.5 \\
{[84.3-99.9]}\end{array}$ & $\begin{array}{l}91.3 \\
{[82.1-99.9]}\end{array}$ & $\begin{array}{l}83.1 \\
{[75.4-91.5]}\end{array}$ & $\begin{array}{l}83.7 \\
{[75.9-92.1]}\end{array}$ \\
\hline
\end{tabular}

Data are sensitivity and specificity and accuracy (\%) followed by $95 \%$ confidence intervals in brackets. Pairwise comparisons between the diagnostic performance of techniques (CTA vs. MRA) and readers (reader 1 vs. reader 2) showed statistically significant differences for all comparisons: diagnostic performance of CTA was statistically significantly higher than MRA for reader 1 and 2 for the evaluation of all, glenoidal or humeral head cartilage lesions for both readers (all $p \leq 0.04$ )

Table 2 Sensitivities, specificities and accuracy (\%) of CTA and MRA in detecting grade 4 cartilage lesions

\begin{tabular}{|c|c|c|c|c|c|}
\hline & & \multicolumn{2}{|l|}{ Reader 1} & \multicolumn{2}{|l|}{ Reader 2} \\
\hline & & CTA & MRA & CTA & MRA \\
\hline \multirow[t]{3}{*}{ All cartilage areas $(n=1,008)$} & Sensitivity & $\begin{array}{l}69.1 \\
{[55.2-80.9]^{*}}\end{array}$ & $\begin{array}{l}41.8 \\
{[28.7-55.9]^{*}}\end{array}$ & $\begin{array}{l}65.5 \\
{[51.4-77.8]}\end{array}$ & $\begin{array}{l}45.5 \\
{[32.0-59.4]}\end{array}$ \\
\hline & Specificity & $\begin{array}{l}99.0 \\
{[98.1-99.5]^{*}}\end{array}$ & $\begin{array}{l}99.5 \\
{[98.8-99.8]^{*}}\end{array}$ & $\begin{array}{l}99.6 \\
{[98.9-99.9]}\end{array}$ & $\begin{array}{l}99.6 \\
{[98.9-99.9]}\end{array}$ \\
\hline & Accuracy & $\begin{array}{l}97.3 \\
{[91.3-99.9]}\end{array}$ & $\begin{array}{l}96.3 \\
{[90.4-99.9]}\end{array}$ & $\begin{array}{l}97.3 \\
{[91.3-99.9]}\end{array}$ & $\begin{array}{l}96.7 \\
{[90.7-99.9]}\end{array}$ \\
\hline \multirow[t]{3}{*}{ Glenoidal cartilage areas $(n=504)$} & Sensitivity & $\begin{array}{l}80.8 \\
{[60.6-93.4]}\end{array}$ & $\begin{array}{l}65.4 \\
{[44.3-82.8]}\end{array}$ & $\begin{array}{l}84.6 \\
{[65.1-95.6]}\end{array}$ & $\begin{array}{l}76.9 \\
{[56.4-91.0]}\end{array}$ \\
\hline & Specificity & $\begin{array}{l}99.0 \\
{[97.6-99.7]}\end{array}$ & $\begin{array}{l}99.6 \\
{[98.5-100.0]}\end{array}$ & $\begin{array}{l}99.4 \\
{[98.2-99.9]}\end{array}$ & $\begin{array}{l}99.4 \\
{[98.2-99.9]}\end{array}$ \\
\hline & Accuracy & $\begin{array}{l}94.1 \\
{[89.6-99.9]}\end{array}$ & $\begin{array}{l}97.8 \\
{[89.4-99.9]}\end{array}$ & $\begin{array}{l}98.6 \\
{[90.1-99.9]}\end{array}$ & $\begin{array}{l}98.2 \\
{[89.8-99.9]}\end{array}$ \\
\hline \multirow[t]{3}{*}{ Humeral head cartilage areas $(n=504)$} & Sensitivity & $\begin{array}{l}58.6 \\
{[38.9-76.5]^{*}}\end{array}$ & $\begin{array}{l}20.7 \\
{[8.0-39.7]^{*}}\end{array}$ & $\begin{array}{l}48.3 \\
{[29.4-67.5]}\end{array}$ & $\begin{array}{l}17.2 \\
{[5.8-35.8]}\end{array}$ \\
\hline & Specificity & $\begin{array}{l}99.0 \\
{[99.2-100.0]^{*}}\end{array}$ & $\begin{array}{l}99.4 \\
{[98.2-99.9]^{*}}\end{array}$ & $\begin{array}{l}99.8 \\
{[98.8-99.9]}\end{array}$ & $\begin{array}{l}99.8 \\
{[98.8-99.9]}\end{array}$ \\
\hline & Accuracy & $\begin{array}{l}96.6 \\
{[88.2-99.9]}\end{array}$ & $\begin{array}{l}94.8 \\
{[86.5-99.9]}\end{array}$ & $\begin{array}{l}91.9 \\
{[83.7-99.9]}\end{array}$ & $\begin{array}{l}92.2 \\
{[84.1-99.9]}\end{array}$ \\
\hline
\end{tabular}

Data are sensitivity and specificity and accuracy (\%) followed by $95 \%$ confidence intervals in brackets

*Pairwise comparisons between the diagnostic performance of techniques (CTA vs. MRA) and readers (reader 1 vs. reader 2) showed statistically significantly better performance of CTA compared to MRA for the evaluation of all cartilage areas, as well as humeral head cartilage lesions for reader 1 (both $p<0.01$ ) (all other $p \geq 0.05$ ) 

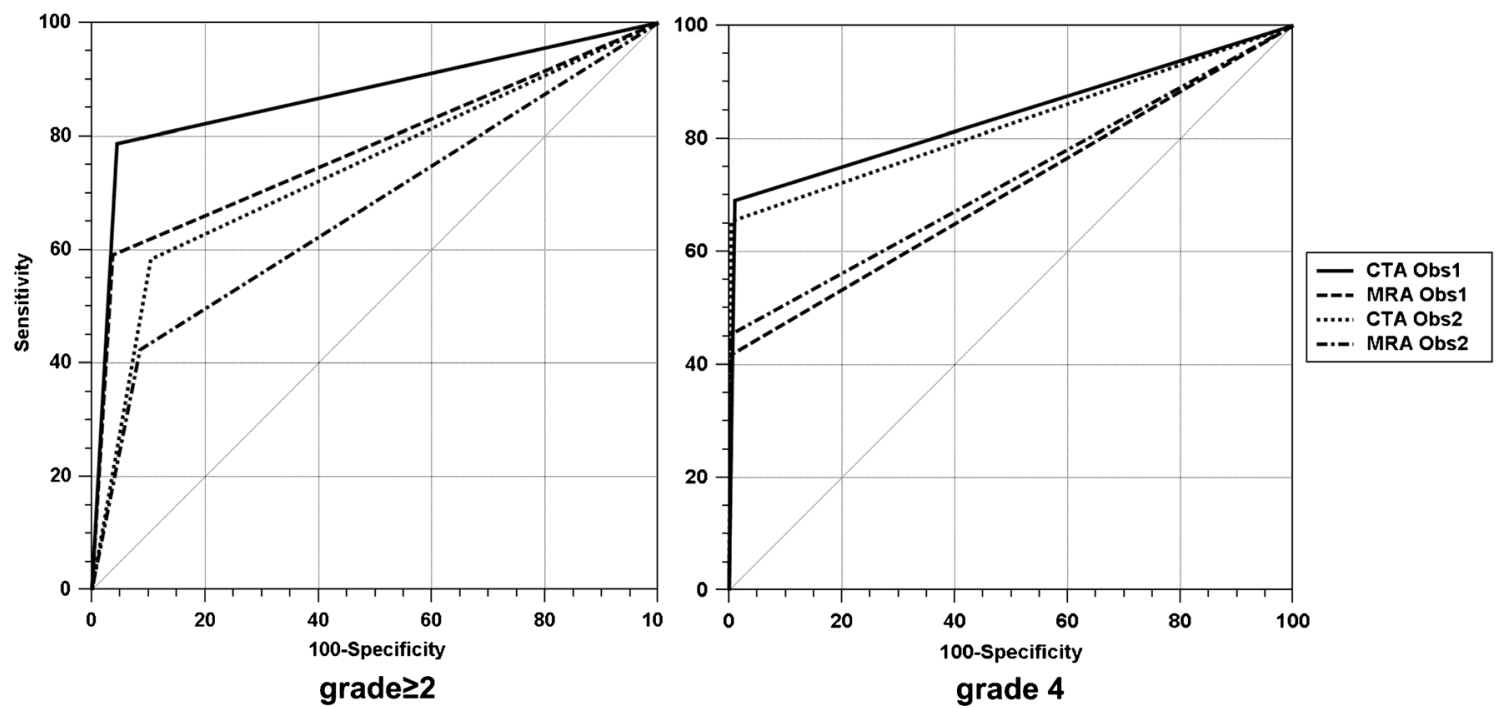

Fig. 6 ROC curves illustrating the comparison of the diagnostic performance of CTA and MRA for the two readers in detecting grade $\geq 2$ and grade 4 cartilage lesions of the shoulder

performance of CTA in a prospective series of 22 patients, showing the high sensitivity and specificity of this technique for the detection of cartilage lesions with substance loss $(80$ $94 \%$ and $92-94 \%$ respectively) [28]. One study by Acid et al., focusing on instability lesions, compared the diagnostic performance of CTA and MRA in assessing glenoid cartilage lesions in the same series of 40 patients [17]. The authors concluded to the better diagnostic performance of CTA (sensitivity $82 \%$, specificity $89 \%$ ) compared to MRA (sensitivity $73 \%$, specificity $94 \%$ ) for the diagnosis of glenoid cartilage lesions in anterior shoulder instability.

Our results are in line with previous authors reporting a moderate performance of MRA for the diagnosis of glenohumeral joint cartilage as well as the better diagnostic performance of CTA. In this regard, this study highlights the importance of a high spatial resolution for the detection of glenohumeral cartilage. Compared to other joints, especially the knee, the lower diagnostic performance of CTA and MRA

Table 3 Interobserver and intraobserver agreement for the presence or absence of cartilage lesions

\begin{tabular}{|c|c|c|c|c|}
\hline & \multicolumn{2}{|c|}{$\begin{array}{l}\text { Interobserver } \\
\text { agreement }\end{array}$} & \multicolumn{2}{|c|}{$\begin{array}{l}\text { Intraobserver } \\
\text { agreement }\end{array}$} \\
\hline & CTA & MRA & CTA & MRA \\
\hline All cartilage areas & $\begin{array}{l}0.63 \\
{[0.56-0.69]}\end{array}$ & $\begin{array}{l}0.54 \\
{[0.46-0.62]}\end{array}$ & $\begin{array}{l}0.95 \\
{[0.92-0.98]}\end{array}$ & $\begin{array}{l}0.86 \\
{[0.80-0.91]}\end{array}$ \\
\hline $\begin{array}{l}\text { Glenoidal cartilage } \\
\text { areas }\end{array}$ & $\begin{array}{l}0.68 \\
{[0.69-0.76]}\end{array}$ & $\begin{array}{l}0.59 \\
{[0.49-0.70]}\end{array}$ & $\begin{array}{l}0.94 \\
{[0.90-0.99]}\end{array}$ & $\begin{array}{l}0.87 \\
{[0.81-0.94]}\end{array}$ \\
\hline $\begin{array}{l}\text { Humeral head } \\
\text { cartilage areas }\end{array}$ & $\begin{array}{l}0.58 \\
{[0.48-0.68]}\end{array}$ & $\begin{array}{l}0.46 \\
{[0.34-0.59]}\end{array}$ & $\begin{array}{l}0.95 \\
{[0.91-0.99]}\end{array}$ & $\begin{array}{l}0.83 \\
{[0.74-0.92]}\end{array}$ \\
\hline
\end{tabular}

Data are kappa coefficients followed by $95 \%$ confidence intervals in brackets in detecting cartilage lesions might be explained by the difference in cartilage thickness (approximately $1.24 \mathrm{~mm}$ for the humeral head and $1.88 \mathrm{~mm}$ for the glenoid compared to over $5 \mathrm{~mm}$ for the patella) [7]. In our study, the higher in-plane resolution with CTA $(0.29-0.39 \mathrm{~mm})$ compared to MRA $(0.63 \mathrm{~mm})$ might explain the better diagnostic performance of the former technique. The better diagnostic performance of CTA and MRA found for the glenoid cartilage compared to the thinner humeral head cartilage also suggests that the thickness of cartilage might play a role.

Several other factors may explain the better diagnostic performance of CTA compared to MRA in this study.

First, the shorter acquisition time of CTA significantly limits motion artefacts compared to MRA, by allowing the $\mathrm{CT}$ acquisition to be performed in one breath hold.

Second, in addition to the in-plane resolution, the throughplane spatial resolution of CTA (0.8 $\mathrm{mm}$ slice thickness) was higher compared to MRA ( $3 \mathrm{~mm}$ slice thickness). Because of the thinness of glenohumeral cartilage this lack of resolution could lead to difficulties in assessing cartilage surface lesions [29]. This is especially true for the humeral head, which is not only thinner but also spherical in shape.

Third, the contrast between the intra-articular iodine and the cartilage at CTA is higher compared to MRA [26, 30, 31].

Fourth, the systematic acquisition of MRA after CTA in our study could have caused resorption of the intra-articular contrast material as well as imbibition of the cartilage, potentially decreasing the overall contrast at cartilage surface. However, a study by Andreisek et al. showed that good quality MRA, including good delineation of cartilage, could be obtained up to $90 \mathrm{~min}$ after the procedure [32].

All these factors may also explain the higher interobserver agreement of CTA compared to MRA. The fact that the interobserver agreement was moderate to substantial with 
the two imaging techniques $(\kappa=0.63$ and 0.54 for CTA and MRA respectively) may be due to a difference in reader experience. However, both readers were musculoskeletal radiologists with previous experience in research in cartilage imaging, suggesting that the difference in interpretation could also be due to inherent difficulties in assessing the glenohumeral cartilage with these techniques.

Compared to previous reports, the strengths of our study include a relatively large series of patients, the evaluation of the diagnostic performance of CTA and MRA performed in the same patients, with prospective arthroscopic correlation. Furthermore, we considered the entire glenohumeral cartilage and divided each articular surface into nine areas for comparison. Most studies evaluating the diagnostic performance of imaging took into account the worst lesion of each articular surface, which might artificially increase diagnostic performance by correlating lesions that might be observed in different areas of the articular surface [3, 4, 8, 17, 27]. Although not perfect, we considered this visual division of the cartilage surface as the best practical way to enable a comparison of the same lesions between imaging and arthroscopy.

Our study has some limitations. First, to avoid compromising patient care, the orthopaedic surgeon was aware of the findings of preoperative imaging, but unaware of the exact topography of the cartilage lesions. Second, we did not obtain any cadaveric correlation, which could represent a better standard than arthroscopy. A cadaveric study would also have the advantage of overcoming the limitation as to the accurate correlation of the topography of cartilage lesions. However, our patient population provided a wider and probably more representative sample of the general population than cadaveric studies, which are generally limited to elderly patients.

Recent review papers in the orthopaedic literature have pointed to the lack of consensus for the therapeutic management of cartilage lesions of the shoulder, and the need for further research in this area [1, 2]. The moderate diagnostic performance of CTA and MRA as shown in this study may limit their value in the setting of such research. More accurate imaging techniques need to be developed for the study of shoulder cartilage, with a focus on improving spatial resolution. Despite recent achievements in dose reduction in musculoskeletal CT, CTA exposes patient to ionizing radiation, so efforts should focus on MRA [33-35]. 3-T MRA with the use of newly developed gradient echo or spin echo based isotropic 3D sequences could be of interest, as well as more specific imaging techniques such as the balanced steady-state free precession (SSFP) techniques, or the use of traction imaging $[6,8,36-39]$.

In conclusion, this prospective study comparing CTA and MRA in the same patients with arthroscopic correlation showed that the diagnostic performance in detecting glenohumeral cartilage lesions was moderate with both techniques, although statistically significantly better with CTA.
Acknowledgments The scientific guarantor of this publication is Patrick Omoumi. The authors of this manuscript declare no relationships with any companies whose products or services may be related to the subject matter of the article. The authors state that this work has not received any funding. No complex statistical methods were necessary for this paper. Institutional review board approval was obtained. Written informed consent was obtained from all subjects (patients) in this study. Some study subjects or cohorts have been previously reported in "Evaluation of rotator cuff tendon tears: comparison of multidetector CT arthrography and 1.5-T MR arthrography" (Radiology 264(3):812-822, 2012. doi:10.1148/radiol.12112062). Methodology: prospective, diagnostic or prognostic study, performed at one institution.

\section{References}

1. Gross CE, Chalmers PN, Chahal J, Van Thiel G, Bach BR, Cole BJ et al (2012) Operative treatment of chondral defects in the glenohumeral joint. Arthroscopy 28:1889-1901

2. Elser F, Braun S, Dewing CB, Millett PJ (2010) Glenohumeral joint preservation: current options for managing articular cartilage lesions in young, active patients. Arthroscopy 26:685-696

3. Guntern DV, Pfirrmann CWA, Schmid MR, Zanetti M, Binkert CA, Schneeberger AG et al (2003) Articular cartilage lesions of the glenohumeral joint: diagnostic effectiveness of MR arthrography and prevalence in patients with subacromial impingement syndrome. Radiology 226:165-170

4. Spencer BA, Dolinskas CA, Seymour PA, Thomas SJ, Abboud JA (2013) Glenohumeral articular cartilage lesions: prospective comparison of non-contrast magnetic resonance imaging and findings at arthroscopy. Arthroscopy 29:1466-1470

5. Rubenstein JD, Li JG, Majumdar S, Henkelman RM (1997) Image resolution and signal-to-noise ratio requirements for MR imaging of degenerative cartilage. AJR Am J Roentgenol 169:1089-1096

6. Gold GE, Reeder SB, Beaulieu CF (2004) Advanced MR imaging of the shoulder: dedicated cartilage techniques. Magn Reson Imaging Clin N Am 12:143-159, vii

7. Yeh LR, Kwak S, Kim YS et al (1998) Evaluation of articular cartilage thickness of the humeral head and the glenoid fossa by MR arthrography: anatomic correlation in cadavers. Skeletal Radiol 27:500-504

8. Dietrich TJ, Zanetti M, Saupe N, Pfirrmann CWA, Fucentese SF, Hodler J (2010) Articular cartilage and labral lesions of the glenohumeral joint: diagnostic performance of 3D water-excitation true FISP MR arthrography. Skeletal Radiol 39:473-480

9. Lecouvet FE, Simoni P, Koutaïssoff S, Vande Berg BC, Malghem J, Dubuc J-E (2008) Multidetector spiral CT arthrography of the shoulder. Clinical applications and limits, with MR arthrography and arthroscopic correlations. Eur J Radiol 68:120-136

10. Omoumi P, Teixeira P, Delgado G, Chung CB (2009) Imaging of lower limb cartilage. Top Magn Reson Imaging 20:189-201

11. Omoumi P, Bafort A-C, Dubuc J-E, Malghem J, Vande Berg BC, Lecouvet FE (2012) Evaluation of rotator cuff tendon tears: comparison of multidetector CT arthrography and 1.5-T MR arthrography. Radiology 264:812-822

12. Rhee RB, Chan KK, Lieu JG, Kim BS, Steinbach LS (2012) MR and CT arthrography of the shoulder. Semin Musculoskelet Radiol $16: 3-14$

13. Hodler J, Kursunoglu-Brahme S, Snyder SJ et al (1992) Rotator cuff disease: assessment with MR arthrography versus standard MR imaging in 36 patients with arthroscopic confirmation. Radiology 182:431-436

14. Zanetti M, Weishaupt D, Gerber C, Hodler J (1998) Tendinopathy and rupture of the tendon of the long head of the biceps brachii 
muscle: evaluation with MR arthrography. AJR Am J Roentgenol 170:1557-1561

15. Bencardino JT, Beltran J, Rosenberg ZS et al (2000) Superior labrum anterior-posterior lesions: diagnosis with MR arthrography of the shoulder. Radiology 214:267-271

16. Jee WH, McCauley TR, Katz LD, Matheny JM, Ruwe PA, Daigneault JP (2001) Superior labral anterior posterior (SLAP) lesions of the glenoid labrum: reliability and accuracy of MR arthrography for diagnosis. Radiology 218:127-132

17. Acid S, Le Corroller T, Aswad R, Pauly V, Champsaur P (2012) Preoperative imaging of anterior shoulder instability: diagnostic effectiveness of MDCT arthrography and comparison with MR arthrography and arthroscopy. Am J Roentgenol 198:661-667

18. Outerbridge RE (1961) The etiology of chondromalacia patellae. J Bone Joint Surg (Br) 43-B:752-757

19. Noyes FR, Stabler CL (1989) A system for grading articular cartilage lesions at arthroscopy. Am J Sports Med 17:505-513

20. Hanley JA, McNeil BJ (1982) The meaning and use of the area under a receiver operating characteristic (ROC) curve. Radiology 143:29-36

21. DeLong ER, DeLong DM, Clarke-Pearson DL (1988) Comparing the areas under two or more correlated receiver operating characteristic curves: a nonparametric approach. Biometrics 44:837-845

22. van Erkel AR, Pattynama PM (1998) Receiver operating characteristic (ROC) analysis: basic principles and applications in radiology. Eur J Radiol 27:88-94

23. Landis JR, Koch GG (1977) The measurement of observer agreement for categorical data. Biometrics 33:159-174

24. Rand T, Brossmann J, Pedowitz R, Ahn JM, Haghigi P, Resnick D (2000) Analysis of patellar cartilage: comparison of conventional MR imaging and MR and CT arthrography in cadavers. Acta Radiol 41: 492-497

25. Li J, Zheng Z-Z, Li X, Yu J-K (2009) Three dimensional assessment of knee cartilage in cadavers with high resolution MR-arthrography and MSCT-arthrography. Acad Radiol 16:1049-1055

26. Gagliardi JA, Chung EM, Chandnani VP et al (1994) Detection and staging of chondromalacia patellae: relative efficacies of conventional MR imaging, MR arthrography, and CT arthrography. AJR Am J Roentgenol 163:629-636

27. Hayes ML, Collins MS, Morgan JA, Wenger DE, Dahm DL (2010) Efficacy of diagnostic magnetic resonance imaging for articular cartilage lesions of the glenohumeral joint in patients with instability. Skeletal Radiol 39:1199-1204

28. Lecouvet FE, Dorzée B, Dubuc JE, Vande Berg BC, Jamart J, Malghem J (2007) Cartilage lesions of the glenohumeral joint: diagnostic effectiveness of multidetector spiral CT arthrography and comparison with arthroscopy. Eur Radiol 17:1763-1771

29. Yeh L, Kwak S, Kim YS, Pedowitz R, Trudell D, Muhle C et al (1998) Anterior labroligamentous structures of the glenohumeral joint: correlation of MR arthrography and anatomic dissection in cadavers. AJR Am J Roentgenol 171:1229-1236

30. Vande Berg BC, Lecouvet FE, Maldague B, Malghem J (2004) MR appearance of cartilage defects of the knee: preliminary results of a spiral CT arthrography-guided analysis. Eur Radiol 14:208-214

31. Hodler J, Loredo RA, Longo C, Trudell D, Yu JS, Resnick D (1995) Assessment of articular cartilage thickness of the humeral head: MRanatomic correlation in cadavers. AJR Am J Roentgenol 165: $615-620$

32. Andreisek G, Duc SR, Froehlich JM, Hodler J, Weishaupt D (2007) MR arthrography of the shoulder, hip, and wrist: evaluation of contrast dynamics and image quality with increasing injection-toimaging time. AJR Am J Roentgenol 188:1081-1088

33. Omoumi P, Verdun FR, Salah YB et al (2014) Low-dose multidetector computed tomography of the cervical spine: optimization of iterative reconstruction strength levels. Acta Radiol 55:335-344

34. Becce F, Ben Salah Y, Verdun FR, Vande Berg BC, Lecouvet FE, Meuli R et al (2013) Computed tomography of the cervical spine: comparison of image quality between a standard-dose and a low-dose protocol using filtered back-projection and iterative reconstruction. Skeletal Radiol 42:937-945

35. Gervaise A, Teixeira P, Villani N, Lecocq S, Louis M, Blum A (2013) CT dose optimisation and reduction in osteoarticular disease. Diagn Interv Imaging 94:371-388

36. Jung JY, Yoon YC, Choi SH, Kwon JW, Yoo J, Choe BK (2009) Three-dimensional isotropic shoulder MR arthrography: comparison with two-dimensional MR arthrography for the diagnosis of labral lesions at 3.0 T. Radiology 250498

37. Kijowski R, Blankenbaker DG, Davis KW, Shinki K, Kaplan LD, De Smet AA (2009) Comparison of 1.5- and 3.0-T MR imaging for evaluating the articular cartilage of the knee joint. Radiology 250 : 839-848

38. Notohamiprodjo M, Horng A, Pietschmann MF et al (2009) MRI of the knee at 3T: first clinical results with an isotropic PDfs-weighted 3D-TSE-sequence. Invest Radiol 44:585-597

39. Becce F, Richarme D, Omoumi P, Djahangiri A, Farron A, Meuli R et al (2012) Direct MR arthrography of the shoulder under axial traction: feasibility study to evaluate the superior labrum-biceps tendon complex and articular cartilage. J Magn Reson Imaging 37: $1228-1233$ 